\title{
NEW-GENERATION LARGE-AREA MUON SCINTILLATION COUNTERS WITH WAVELENGTH SHIFTER FIBER READOUT FOR CDF II
}

\author{
A. Artikov ${ }^{1}$, J.Budagov, I. Chirikov-Zorin, D. Chokheli ${ }^{2}$, \\ V. Kolomoets, M.Lyablin, O.Pukhov, A. Sissakian \\ Joint Institute for Nuclear Research, Dubna
}

\section{G. Bellettini, A. Menzione, A. Scribano}

INFN and University of Pisa, Italy

\section{G. Pauletta}

INFN and University of Udine, Italy

\author{
B. Grinyov, V. Lebedev, V. Lyubynskiy, V. Senchyshyn \\ Institute of Scintillating Materials, Kharkiv, Ukraine
}

V. Kovtun, I. Zaljubovsky

Kharkiv Karazin National University, Ukraine

\section{S. Tokar}

Comenius University, Bratislava, Slovakia

\section{N. Giokaris, A.Manousakis-Katsikakis \\ University of Athens, Greece}

\begin{abstract}
New scintillation counters have been designed and constructed for upgrading of the CDF detector at the Fermilab Tevatron. A novel light collection technique using wavelength shifting fibers, together with a high-quality polystyrene-based scintillator UPS 923A, has resulted in compact counters with good and stable light collection efficiency over their lengths extending up to $320 \mathrm{~cm}$. Design, construction and performance of counters are presented. Properties of the fibers and the scintillator, such as light output, light attenuation, decay time and long-term stability, are investigated. It is found that the polystyrenebased scintillator, unlike the polyvinyltoluene-based one, has better properties adequate for long-term experiments.
\end{abstract}

Разработаны и созданы новые сцинтилляционные счетчики для модернизации детектора CDF на тэватроне FNAL. Оригинальная техника светосбора с применением спектросмещающих волокон совместно с высококачественным сцинтиллятором UPS 923А на основе полистирола позволила создать компактные счетчики (длиной вплоть до $320 \mathrm{~cm}$ ) с хорошей и стабильной эффективностью светосбора. Представлены конструкция и характеристики счетчиков. Исследованы свойства волокон и сцинтиллятора, такие как световыход, ослабление света, время высвечивания и долговременная стабильность. Обнаружено, что сцинтиллятор на основе полистирола имеет лучшие свойства, отвечающие требованиям для долговременных экспериментов, чем сцинтиллятор на основе поливинилтолуола.

\footnotetext{
${ }^{1}$ On leave from NPL, Samarkand State University, Uzbekistan.

${ }^{2}$ On leave from HEPI, Tbilisi State University, Georgia.
} 


\section{INTRODUCTION}

The importance of detecting muons at the Collider Detector at Fermilab (CDF) and of measuring their momentum accurately can hardly be overstressed. Drell-Yan muon-pair production is a mean for testing the basics of the EW theory and for searching for additional vector bosons, leptons, quark substructure, and possible extra dimensions. Muons originate with large branching fractions from the decay of the top quark and beauty-flavored hadrons. Detailed studies of production and decay properties of the top quark are eagerly awaited and can be best performed in the muon decay channels. An extended beauty physics program, ranging from relatively simple lifetime studies to the study of subtle $C P$-violation effects, can be performed on a muon event sample. A search for the light Higgs boson, which is expected to decay predominantly into $b$-quark pairs, can be made in the muon $b$-decay channel. Finally, muons are expected to appear as products of the decay chain in most SUSY processes [1].

A considerable effort was therefore made to upgrade the CDF for increasing the muon acceptance [1]. As a result, the upgraded CDF detector, which started a new run of data taking (Run 2) in March 2001, features nearly complete muon coverage up to a pseudorapidity $\eta$ of \pm 1.5 .

Over most of the solid angles the muon detector is a sandwich of drift chambers and plastic scintillation counters, which can be used to trigger on penetrating muon tracks to identify their bunch crossing and to signal their trajectory. In particular, the fast response of the scintillation counters is important in associating a muon track with its corresponding bunch crossing.

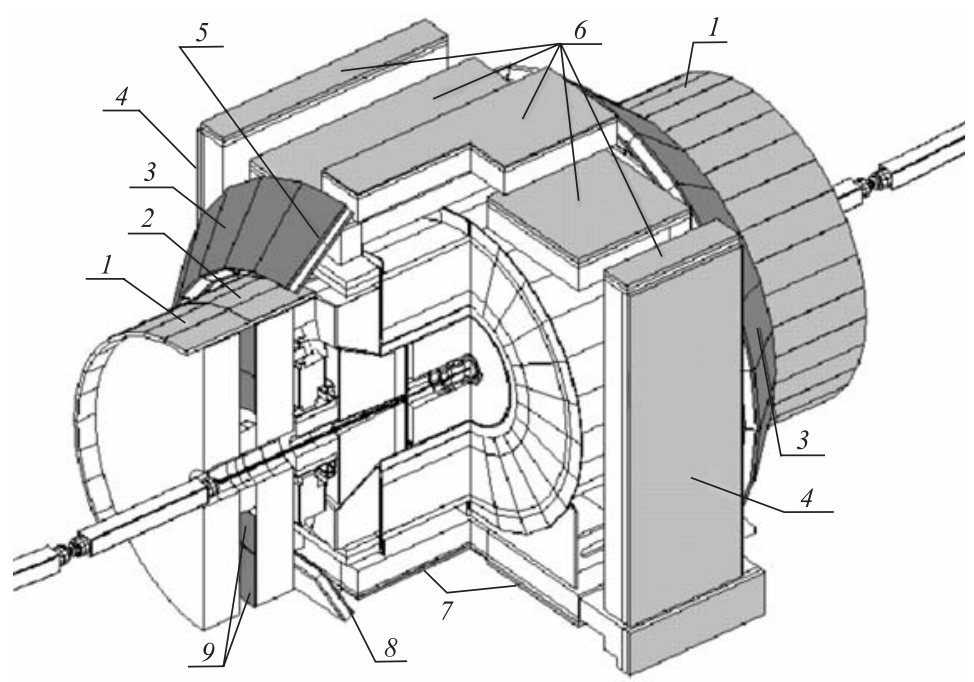

Fig. 1. CDF II detector: $1-$ BSU rear; $2-$ BSU forward; 3 - CSX external; 4 - CSP wall; 5 CSX internal; $6-$ CSP top; $7-$ CSP bottom; $8-$ CSX miniskrit; $9-$ TSU

The complex and compact CDF geometry (see Fig. 1 and Ref. [1]) required construction and installation of a large set of counter hodoscopes employing a total of about 1200 plastic 
scintillators with different dimensions and light collection assemblies. Part of those (more than 600 counters) constructed for the CDF upgrade were designed to overcome the space restrictions and to improve light collection from long counters.

This paper describes the design and performance of these new counters distinguished by their unconventional and compact light collection system.

\section{COUNTERS FOR CDF II}

Muon momenta are measured in CDF by the central tracker immersed in a solenoid magnetic field. However, a key signature is obtained from dedicated muon chambers and counters which are placed behind the calorimeter so as to exploit the ability of the muon to traverse thick absorbers. Counter areas are large in order to keep the number of individual elements within a manageable limit. Conventional collection systems rely on plexiglas light guides mounted on the counter extremities. These light guides are large and often conflict with other detector parts. Because of this difficulty, the coverage of the Run 1 muon detector was incomplete [1]. Light collection through the counter extremities also involves a large optical path length in the scintillator and is therefore susceptible to deterioration in the scintillator properties. This problem seriously affected the performance of the Run 1 counters [2]. The new counters for Run 2 were designed to overcome any potential performance degradation.

Locations of the CDF II muon detectors are shown in Fig. 1. There are in total about 1200 muon scintillation counters in the CDF II detector. The longest of these counters $(320 \mathrm{~cm})$ cover the central region $(|\eta|<0.6)$ and are named Central Scintillator uPgrade (CSP) counters. They are laid on the outside of the drift chamber of the Central Muon uPgrade (CMP) chamber stacks, behind $61 \mathrm{~cm}$ of steel shielding located outside the central calorimeter. The new CSP counters cover the top and bottom of the detector (see Fig. 1). The original counters cover the north and south walls.

Because of deterioration of their transmission properties, the older scintillators were refurbished [2] by the addition of a light collection system matching the new counter design.

The scintillation counters of the Central Scintillator eXtension (CSX) are arranged in a conical hodoscope to cover the region $0.6<|\eta|<1.2$. The CSX azimuthal coverage was also completed in the course of upgrading by addition of two $90^{\circ}$ sectors (the «miniskirts») in the lower parts of both the east and west cones and a $30^{\circ}$ sector (the «keystone») in the upper part of the west cone. These counters of conventional design were designed and constructed for Run 1, but their installation was delayed by several obstructions [3]. The light collection system of a subset of them had to be modified according to the new design.

To extend the muon coverage to $|\eta|=1.5$, two overlapping barrel hodoscopes of $160 \mathrm{~cm}$ long scintillators, called the Barrel Scintillator Upgrade (BSU), were constructed to cover a barrel of drift chambers, called the Barrel Muon Upgrade (BMU), which surround the steel of the former toroid magnets (now decommissioned and used for shielding), for about $3 / 4$ of the azimuth (see Fig. 1). The bottom of the toroid steel is obstructed by its supports. Finally, a pinwheel counter hodoscope, called the Toroid Scintillator Upgrade (TSU), is located between the toroids and covers the region from $|\eta| \approx 1.2$ to $|\eta| \approx 1.5$. Together with the outer BSU ring, these counters form a projective trigger for the BMU in the region. The TSU counters were constructed at Michigan State University according to a different design [4]. 
The design, construction and initial performance of the CSP and BSU counters constructed for the CDF upgrading are described below. A major improvement in the design of the new counters was achieved by employing ribbons of wavelength shifting (WLS) fibers suitably coupled to the sides of the plastic bars for optimal light collection. Bulky light guides were thus avoided and light could be collected more uniformly and more efficiently from the scintillating material. Light collection from plastic scintillators by means of WLS fibers were adopted in several sandwich calorimeters [5]. This application is different because of its much higher light collection efficiency. In our trigger counters, it was vital to reach full efficiency on traversing Minimum Ionizing Particles (MIP). In calorimeters, the amount of light collected from each scintillator layer is not as critical since the detector response is the sum of the signals from a large number of layers.

\section{THE R\&D PHASE}

2.1. Merits of the Fiber Readout. It is customary to collect light from plastic scintillator bars from the short side of the bar with plexiglas light pipes, which are either of a «fish tail» design or an assembly of suitably bent plexiglas strips. However, since the plastic scintillator bars of the CDF II muon detectors are up to $320 \mathrm{~cm}$ long and their transverse dimensions are relatively small ( $\leqslant 30 \mathrm{~cm}$ wide and $\leqslant 2 \mathrm{~cm}$ thick), the light collection through conventional light pipes in counters of this geometry creates several problems:

- The light transmission efficiency in the scintillator is limited by the nonperfect transparency of the bulk material and by the internal reflection efficiency. In our counters both the optical path length and the number of reflections are large. It follows that the plastic surface must be accurately polished to increase the internal reflection coefficient and that the bulk transparency must be extremely good. Furthermore, since the counters will be used for many years, both these properties must be time and radiation-resistant. Even small deterioration of the bulk transparency or the surface quality leads to large reduction in the amount of light which reaches the photomultiplier (PMT).

- For efficient light collection the area of the PMT photocathode must be as large as that of the scintillator surface which is optically coupled to the light pipe. Since the latter can be as large as $60 \mathrm{~cm}^{2}$, large and expensive PMTs would be required for optimal light collection. Moreover, large phototubes are particularly sensitive to stray magnetic fields.

- Large light guides and large PMTs occupy a lot of space, and overlaps and conflicts with other detectors can be generated.

These problems were addressed and solved to a large degree by the new CDF counter design, which is based on the use of WLS fibers for light collection. This collection method was used for all new counters with mirror differences depending on the counter shape. This is schematically shown in Fig. 2.

The merits of this method are:

- Light can be collected from counters of any shape with no concern for possible obstructions by light pipes. It is therefore possible for hodoscopes to obtain complete coverage even in complicated geometries and when one is short of space.

- Since light is collected from the long edge of the scintillator bar, the number of multiple reflections suffered by the light before exiting the edge of the bar is much reduced with respect to conventional light pipes, particularly where the ratio of the transverse to the longitudinal 


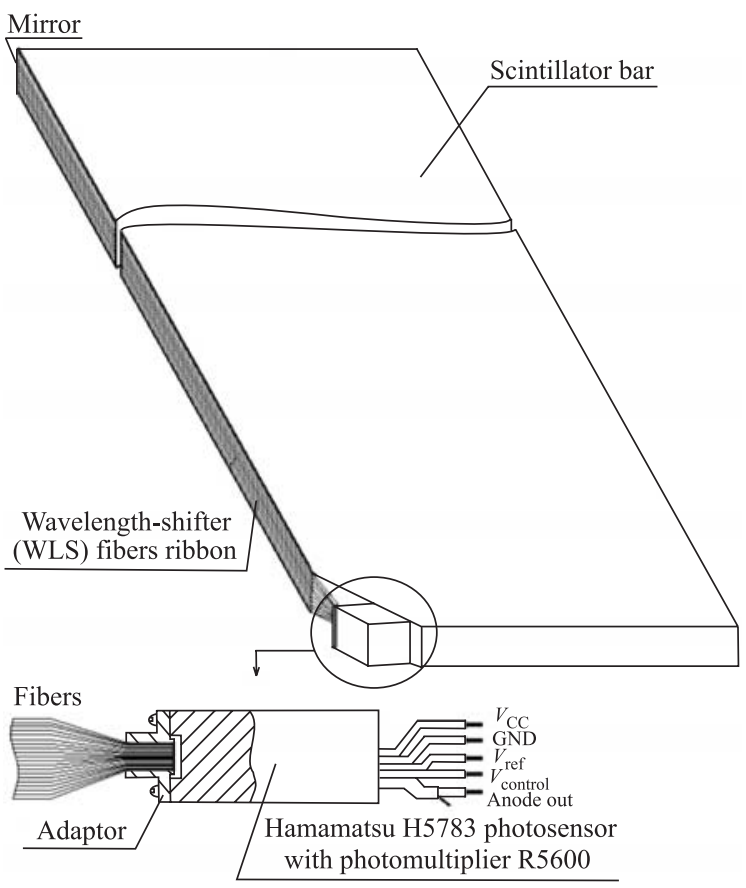

Fig. 2. Scheme of the counter employing a WLS fiber light guide

dimensions is small. As a result, the requirement to the quality of bar surface polishing is weakened. It also follows that good transparency and light yield of the bulk scintillator become less critical and that scintillator aging and radiation damage can be better tolerated. The overall result is a more reliable and durable detector.

- One is no longer subject to Liouville's theorem and the total cross section of the WLS fibers comprising the light-collecting ribbons, which must be optically connected to the PMT photocathodes, is small $\left(\sim 0.5 \mathrm{~cm}^{2}\right.$ in our case). Small PMTs can therefore be used and the detector can be made more compact with significant cost saving.

- Small PMTs are less sensitive to magnetic fields. Magnetic shields are often not required or can be much simpler and less expensive.

- The labor costs are greatly reduced with respect to those of light-guide fabrication. When WLS fibers are used, one can glue the fiber ribbons to the scintillator and obtain a mechanically solid counter with a relatively simple assembly procedure.

- In some cases, multi-anode PMT can be used to read out several counters with a corresponding reduction in the cost of the electronic instrumentation.

2.2. Manufacturing and Testing of Counter Prototypes. A number of prototypes of different shapes and sizes were built [6-10] to test the viability of collecting light by means of WLS fiber ribbons as outlined above and their light yield was measured.

Our study addressed the expected performance of counters with geometries corresponding to the BSU, CSP and $\mathrm{WSU}^{1}$ counters. The BSU prototype was a rectangular scintillator

\footnotetext{
${ }^{1}$ The WSU counters were originally intended for the back of the wall calorimeters and were similar to TSU counters, but on the basis of later CDF collaborators considerations they were not built.
} 
$180 \mathrm{~cm}$ long, $17 \mathrm{~cm}$ wide and $2 \mathrm{~cm}$ thick. The CSP prototype was $300 \mathrm{~cm}$ long, $30 \mathrm{~cm}$ wide and $2 \mathrm{~cm}$ thick. The trapezoidal WSU counter prototype was $180 \mathrm{~cm}$ long and $1.5 \mathrm{~cm}$ thick with bases of lengths 30 and $40 \mathrm{~cm}$. All bars were made of scintillator UPS 923 $\mathrm{A}^{1}$ [11].

Counters were wrapped up in aluminized paper for diffuse internal light reflectivity and backed by black plastic foil for outside light tightness. An absolute calibration of the PMT (response to single photoelectron (ph.e.)) [12] was obtained by flashing the photocathode with a fast light-emitting diode (LED). The response of each counter in terms of ph.e./MIP was measured by using cosmic muons which were selected by a small counter telescope $(4 \times 7 \mathrm{~cm})$.

The PMT was joined to the fiber ribbon bundle by optical grease. The other end of the fiber ribbon was blackened in the first set of measurement, then grinded flat with sand paper and a small piece of aluminum foil was applied to it with optical glue. This very simply technique provided a reflectivity of about $60 \%$. The most relevant results of the tests are shown in Fig. 3.

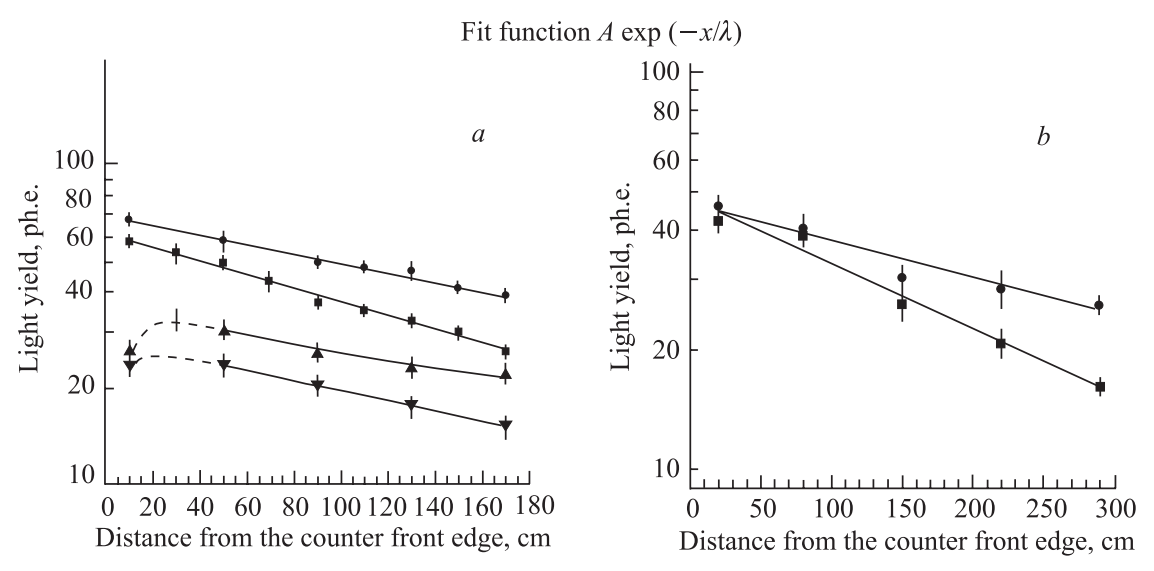

Fig. 3. Test results for: $a)$ the BSU $(180 \times 17 \times 2 \mathrm{~cm})$ counter prototype: - - ends of fibers are aluminized; $A=(69.1 \pm 2.6)$ ph.e.; $\lambda=(293 \pm 28) \mathrm{cm} ; \mathbf{\square}-$ ends of fibers are blackened; $A=(61.2 \pm 2.0)$ ph.e.; $\lambda=(201 \pm 13) \mathrm{cm}$; the WSU $(180 \times 40(30) \times 1.5 \mathrm{~cm})$ counter prototype: $\boldsymbol{\Delta}$ - ends of fibers are aluminized; $A=(34.6 \pm 1.4)$ ph.e.; $\lambda=(347 \pm 48) \mathrm{cm} ; \boldsymbol{\nabla}$ - ends of fibers are blackened; $A=(27.7 \pm 1.8)$ ph.e.; $\lambda=(275 \pm 40) \mathrm{cm}$; and $b)$ the CSP $(300 \times 30 \times 2 \mathrm{~cm})$ counter prototype: - ends of fibers are aluminized; $A=(46.7 \pm 2.5)$ ph.e.; $\lambda=(473 \pm 59) \mathrm{cm}$; $\mathbf{\square}$ - ends of fibers are blackened; $A=(47.8 \pm 2.6)$ ph.e.; $\lambda=(268 \pm 17) \mathrm{cm}$. The light yields as a function of the distance to PMT

In contrast to the scintillator the WLS fibers are rather transparent for their own light emitted. Therefore, the effective attenuation length in such counters basically depends on the fiber transparency. The scintillator transparency is of lower importance.

The effective light attenuation of the WSU prototype $(\lambda \approx 350 \mathrm{~cm})$ is larger than for the BSU prototype $(\lambda \approx 290 \mathrm{~cm})$. The WSU counter light yield is twice smaller than the one of

\footnotetext{
${ }^{1}$ Ukrainian Plastic Scintillator 923A is manufactured by the Institute of Scintillating Materials, Kharkiv, Ukraine.
} 
the BSU (see Fig. 3, the $A$ parameter), instead of expected $\approx 1.3$ times, due to the difference in bar thickness: WSU $-1.5 \mathrm{~cm}$; BSU $-2.0 \mathrm{~cm}$. This discrepancy is caused by larger scintillator light absorption in the WSU counter, because the WSU bar is thinner and wider than the BSU one.

Thus, in the WSU counter light collection is essentially realized by the light propagation in the fibers, whereas in the BSU light propagation takes place in both the fibers and the scintillator. Therefore, in the WSU prototype an effective attenuation length basically depends on fiber properties and is larger as fibers have a longer light attenuation length in comparison to that of the scintillator bar.

The Y11 dopant [13] feature is partial overlap of the emission and absorption spectra. Therefore, the reabsorption effect shifts the emission spectrum to the longer wavelength region, where absorption is less significant while photons are propagating in the fibers. The partial reabsorption process increases the effective attenuation length in long counters relative to shorter ones. Therefore, the effective attenuation length in the CSP prototype $(\lambda \approx 470 \mathrm{~cm})$ is larger than in the BSU counter $(\lambda \approx 290 \mathrm{~cm})$.

\section{SCINTILLATOR PROPERTIES}

The quality of scintillator counters is determined primarily by the quality of the scintillating material used by the manufacturer. The scintillator type that was eventually chosen is UPS 923A. It was produced in 1997-2000 at the Institute of Scintillating Materials in Kharkiv under the supervision of JINR Dubna personnel. The bulk material is polystyrene doped with $2 \%$ PTP and $0.03 \%$ POPOP. The light output is $50 \%$ relative to anthracene.

3.1. Light Attenuation. In 1992 the 2-m-long scintillation counters employing UPS 923A and NE $114^{1}$ plastic scintillators viewed by a single photomultiplier at the short side of the bars through a «fish tail» light guide were tested by using cosmic muons. The light yield of UPS 923A was found to be about $25 \%$ higher than that of NE 114, while the Technical Attenuation Length (TAL) was approximately the same [11]. Note that the TAL of a plastic scintillation bar is defined as the length required to reduce the light signal by a factor $e$ and depending upon bulk transmission of the scintillator, thickness and shape of the bar and reflective properties of the surface.

The scintillator material transparency is determined by the very often used parameter the so-called Bulk Attenuation Length (BAL); this is a length reducing light by a factor $e$. The BAL of the UPS 923A scintillator was measured by means of a cadmium-vapor laser with a $441 \mathrm{~nm}$ wavelength and it was about $260 \mathrm{~cm}^{2}$. It is important to note that the main optical parameter of the scintillator material is transparency for their own light emitted.

The BAL of the scintillator for its own light emitted was measured by the parallel beam light with a spectrum very close to the emission spectrum of the scintillator (spectrum of the POPOP wavelength shifter) [9]. An ordinary filament lamp was used as a source of light. The light spectrum of the lamp was corrected by the «CC5» and «ФC1» light filters $[15,16]$. The spectrum of the parallel beam light was checked by the optical spectrum analyzer.

\footnotetext{
${ }^{1} \mathrm{NE} 114$ was manufactured by Nuclear Enterprises Ltd. This product is equivalent to BC 416 , manufactured by Bicron Corp.

${ }^{2}$ The BAL of NE 110 scintillator for the $458 \mathrm{~nm}$ wavelength is $204 \mathrm{~cm}$ [14].
} 
The POPOP dopant features partly overlapping of the absorption and emission spectra [17]. Therefore the short-wavelength part (less than about $400 \mathrm{~nm}$ ) of the emitted light of the scintillator is attenuated very rapidly basically due to reabsorption effect. Thus the spectrum of the emitted light is shifted to the long-wave region, where the processes of absorption and reabsorption are less significant $[14,18]$. In general, therefore, the light attenuation of the scintillator should not exhibit exponential dependence upon the distance of light propagation. To investigate self-absorption, we measured the BAL of a set of the UPS 923A scintillator samples with different lengths made from the same scintillation block (Fig.4).

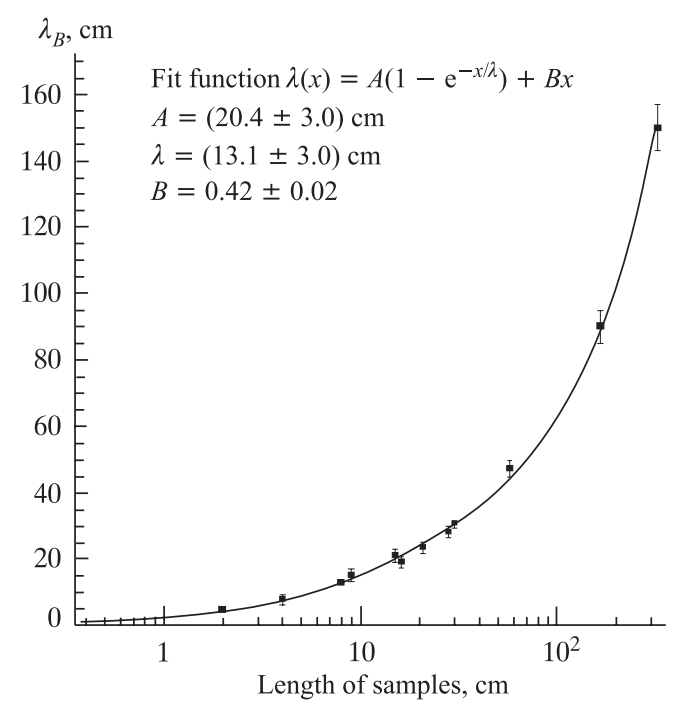

Fig. 4. Dependence of the bulk attenuation length $\lambda_{B}$ on the light propagation in the scintillator

The experimental data were approximated by the function

$$
\lambda_{B}=A\left(1-\mathrm{e}^{-x / \lambda}\right)+B x
$$

The first term of the expression describes strong self-absorption of scintillator light in the overlap region. The BAL for the light that traveled less than about $30 \mathrm{~cm}$ depends upon the concentration and properties of the fluors, wavelength shifter ${ }^{1}$ and scintillator base material. The second term describes the region (more than $30 \mathrm{~cm}$ ), where the short-wavelength wing of the spectrum of emitted light was essentially absorbed and therefore the bulk transmission of the scintillation material is increasing. In this region the BAL becomes dependent on the base material and dopant quality (purity).

The number of the scintillation bars required for the upgrade of the CDF II muon system is more than 600 . The above-described BAL measurement method was developed and used for quality control of the scintillation bar material production.

\footnotetext{
${ }^{1}$ The scintillator with a large Stokes shift wavelength shifter has comparatively small self-absorption of its emitted light [17].
} 


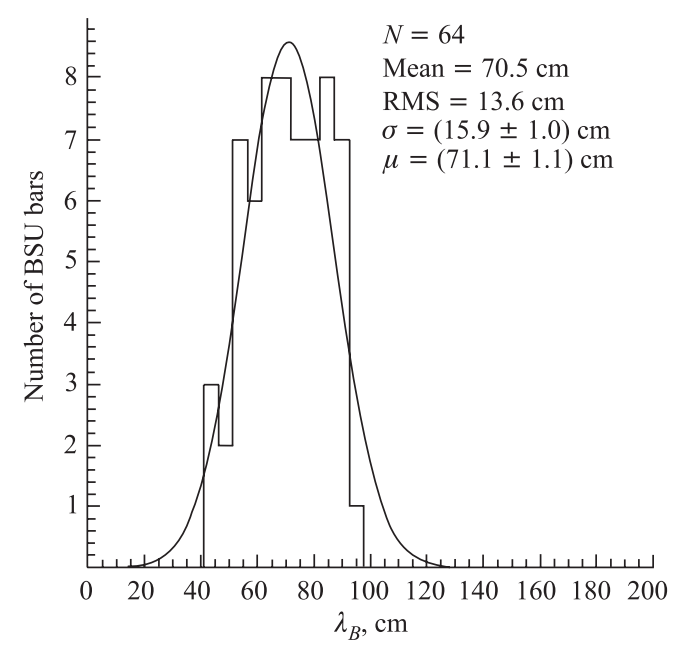

Fig. 5. Distribution of the bulk attenuation length for one lot of BSU bars

For example, Fig. 5 shows the BAL distribution for one lot of BSU bars measured for the long side $163.8 \mathrm{~cm}$ (see Sec. 4).

3.2. Time Response Properties of the Scintillator and WLS Fibers. High-resolution fast coincidence systems to observe rare particles against a large background of other events and time-of-flight measurements are needed in many experiments [19]. The scintillator detector time resolution is determined primarily by the shape of the light pulse excited by a particle in the scintillator and also by time properties of the WLS fibers used in the optical readout system.

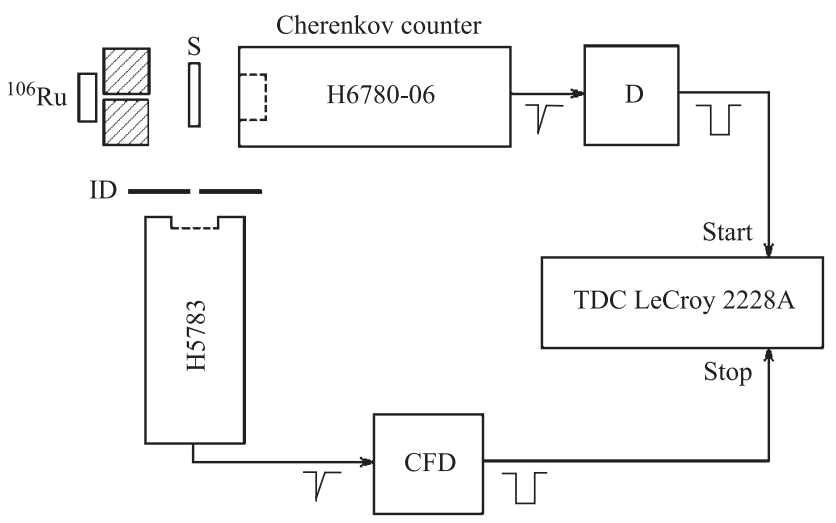

Fig. 6. Experimental setup for decay time measurements

The light pulse shape studies were performed by means of the single-photon method of Bollinger and Thomas [20]. Figure 6 shows a simplified block diagram of the time spectrometer we invented for decay time study. Investigation samples (S) were disks $15 \mathrm{~mm}$ in diameter and $1.5 \mathrm{~mm}$ thick with polished surfaces. The samples were excited by electrons from a ${ }^{106} \mathrm{Ru}+{ }^{106} \mathrm{Rh}$ source with maximum energy 3.54 MeV. To improve the time resolution 
of the setup, a Cherenkov counter was used to generate a trigger signal. The Cherenkov radiator cylinder of diameter $9 \mathrm{~mm}$ and height $6 \mathrm{~mm}$ was made of quartz. The radiator was coupled to the Hamamatsu H6780-06 photosensor (sensitive in ultraviolet region) by optical grease RHODORSIL SI 200. The Cherenkov counter signal was discriminated by the leading edge discriminator (D) and an output signal was used to start the LeCroy 2228A TDC.

The H5783 photosensor was used to detect a single photon from the sample edge. The photon counter was tuned to the single-photon mode with the help of an iris diaphragm (ID), which reduced the detected signal to approximately 0.01 photoelectrons on average. Such a radiation attenuation is needed to maintain the probability of detection of two or more photoelectrons in one light pulse at a negligibly small level $\left(<10^{-4}\right)$. The single-photon signal from H5783 is discriminated by a constant fraction discriminator (CFD) and an output signal is used to stop the TDC.

The time resolution of the system for a single photoelectron was measured with lucite samples which yield fast Cherenkov light signals. Since the light from the Cherenkov process is emitted essentially instantaneously and the light output was limited to a single photoelectron, the measured time distribution is a direct measure of the system response (apparatus function). Figure 7 shows a typical Cherenkov time distribution. In the Gaussian approximation the measured system resolution is $\sigma \approx 150 \mathrm{ps}$.

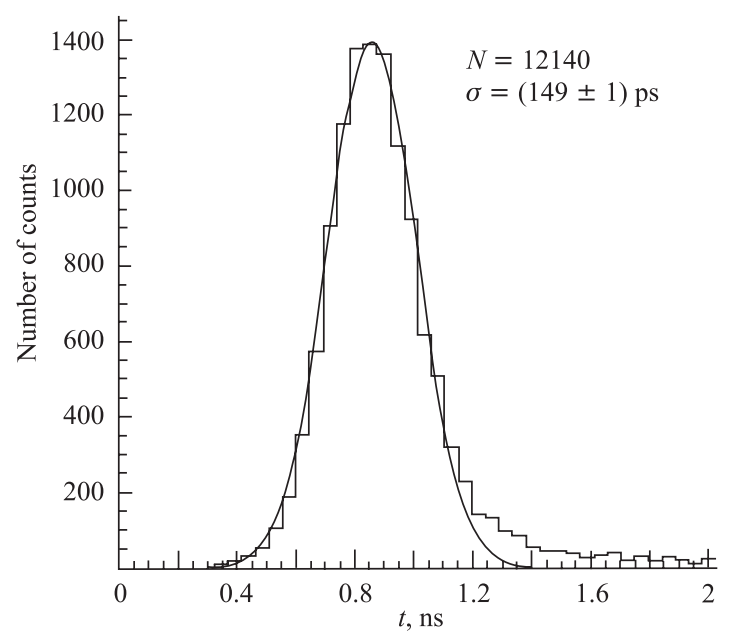

Fig. 7. Apparatus function measurement with Cherenkov light

Many processes contribute to scintillation light emission [21, 22]. The light pulse shape $f(t)$ from plastic scintillators can be described as consisting of a fast component generated by a two-step scintillation cascade and an additional slow component; with these assumptions, $f(t)$ is expressed as follows:

$$
f(t)=\frac{N}{1+R}\left[\frac{\mathrm{e}^{-t / \tau_{2}}-\mathrm{e}^{-t / \tau_{1}}}{\tau_{2}-\tau_{1}}+R \frac{\mathrm{e}^{-t / \tau_{3}}}{\tau_{3}}\right],
$$

where $N$ is the total number of photons; $\tau_{1}$ is the rise time constant; $\tau_{2}$ is the decay time constant of the fast scintillation process; $\tau_{3}$ is the decay time of the slow component, and $R$ is the ratio of the light components produced through the slow and fast decay processes. 


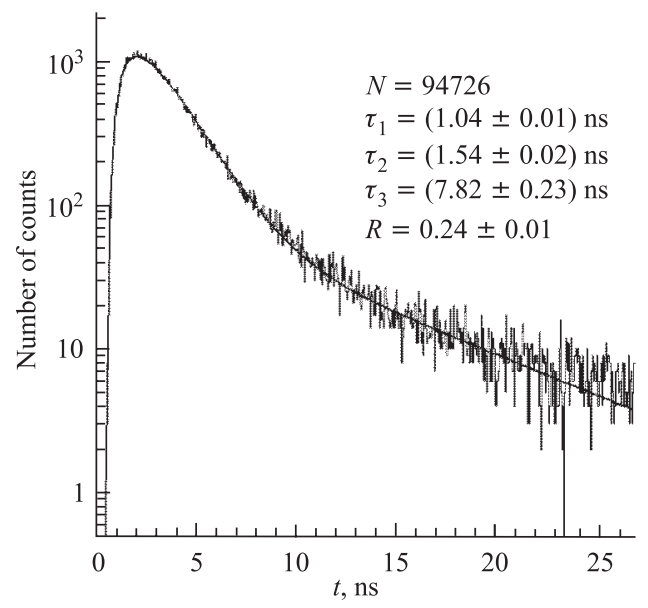

Fig. 8. Time distribution spectrum of the light pulse from the UPS 923A scintillator sample

Figure 8 shows the time spectrum of the light pulses of the UPS 923A scintillator. The measured time distribution is described by convolution integral of expression (2) and the Gaussian apparatus function (Fig. 7). But in our case the time resolution of the system for single photoelectrons (FWHM $\approx 350 \mathrm{ps}$ ) is significantly smaller than the scintillation pulse duration. Therefore, in the first approximation the spectrum was fitted by using formula (2). A comparison of the measured time distribution with the results of the fit is shown in Fig. 8 .

We studied the time response of WLS fibers. For this purpose the UPS 923A scintillator samples were coupled to WLS fibers with optical glue. Figures 9 and 10 present the time

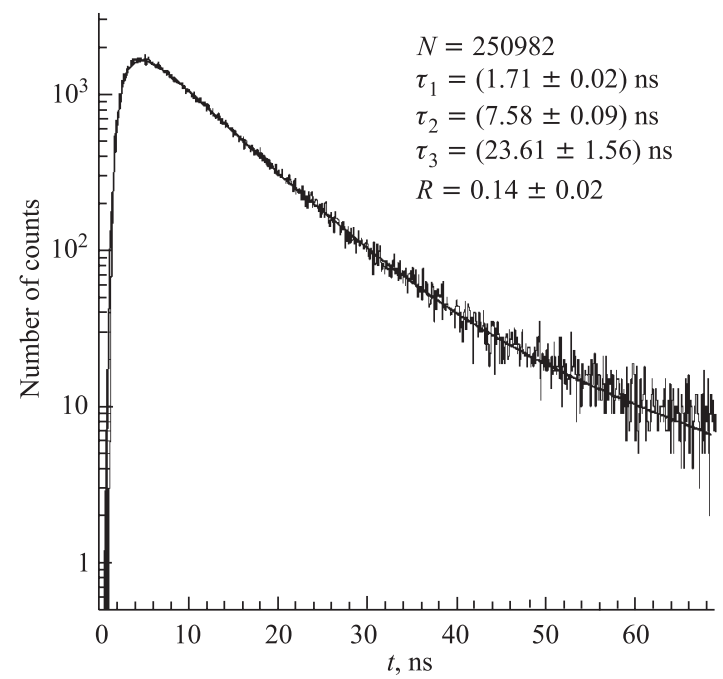

Fig. 9. Time distribution spectrum of the light pulse from the UPS 923A sample with Y11(250) MS fibers 


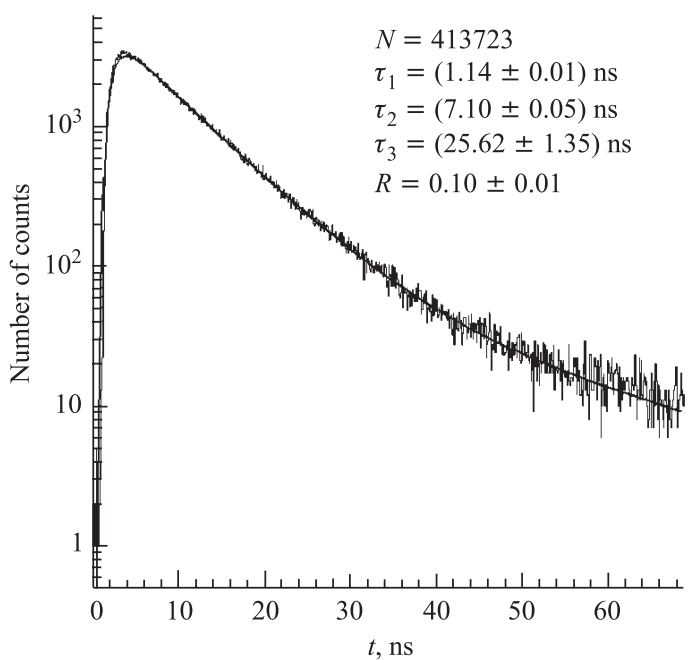

Fig. 10. Time distribution spectrum of the light pulse from the UPS 923A sample with K27(200) MS fibers

spectrum of the light pulse from UPS 923A with WLS fibers. The time spectra were fitted by using phenomenological formula (2) as well.

To conclude, we list the main useful time parameters of light pulse shapes of a scintillator and WLS fibers: rise time (level 0.1-0.9), fall time (level 0.9-0.1), FWHM (level 0.5-0.5) and pulse duration (level $0.1-0.1$ ) as given in Table 1 . The parameters of the Cherenkov light pulse from the Lucite samples are given in Table 1 as well. It is important to note that the rise time of the light pulses from the UPS 923A scintillator with WLS fibers is fast $(\approx 2 \mathrm{~ns})$; therefore, counters with WLS fiber readout can be used in fast coincidence systems and time-of-flight measurements.

Table 1. Measured time parameters of the light pulse shapes

\begin{tabular}{|l|c|c|c|c|}
\hline \multicolumn{1}{|c|}{ Material } & $\begin{array}{c}\text { Rise time, ns } \\
\text { (level 0.1-0.9) }\end{array}$ & $\begin{array}{c}\text { Fall time, ns } \\
\text { (level 0.9-0.1) }\end{array}$ & $\begin{array}{c}\text { FWHM, ns } \\
\text { (level 0.5-0.5) }\end{array}$ & $\begin{array}{c}\text { Pulse duration, ns } \\
\text { (level 0.1-0.1) }\end{array}$ \\
\hline UPS 923A & 0.8 & 5.3 & 3.0 & 7.2 \\
UPS 923A + K27 & 1.7 & 17.3 & 7.8 & 19.1 \\
UPS 923A + Y11 & 2.2 & 18.0 & 8.8 & 22.4 \\
Lucite & 0.23 & 0.23 & 0.33 & 0.65 \\
\hline
\end{tabular}

3.3. Long Term Stability. The CDF II detector must operate for about 10 years. Therefore, the problem of ensuring stability and full efficiency of the muon scintillation counters over that period of time is serious. For this reason it is necessary to study natural degradation of the properties of the scintillator bars. Natural scintillator aging depends on the components used. We investigated natural aging of the plastic scintillator UPS 923A based on polystyrene (PS) and compared it to a well-known product NE 114 based on polyvinyltoluene (PVT). For these studies two long scintillation counters with the UPS 923A and NE 114 plastic scintillators 
viewed by a single photomultiplier (EMI 9814B) at the short side of the bars through a «fish tail» light guide were built. The scintillator itself was a $200 \times 30 \times 2 \mathrm{~cm}$ bar. At one end a «fish tail» light guide was glued to the bar edge by special optical cement NE 581 . The second end was painted black to suppress light reflection. The detailed description of the design of the counters is given in [11].

The properties of the counters were investigated with cosmic muons selected by a telescope of two small scintillation counters $(\sim 4 \times 10 \mathrm{~cm})$. The counter to be studied was placed between them. Moving the telescope along the counter axis, we measured dependence of the light yield on the distance from the bar edge. A LeCroy ADC 2249A charge-digital converter measured the PMT signal amplitude. The spectrometric channel was calibrated in absolute units, i.e., in the number of photoelectrons created on the PMT photocathode. The calibration was done with a fast LED by using low-intensity light flashes. The calibration method and measurements are detailed in $[11,23]$.

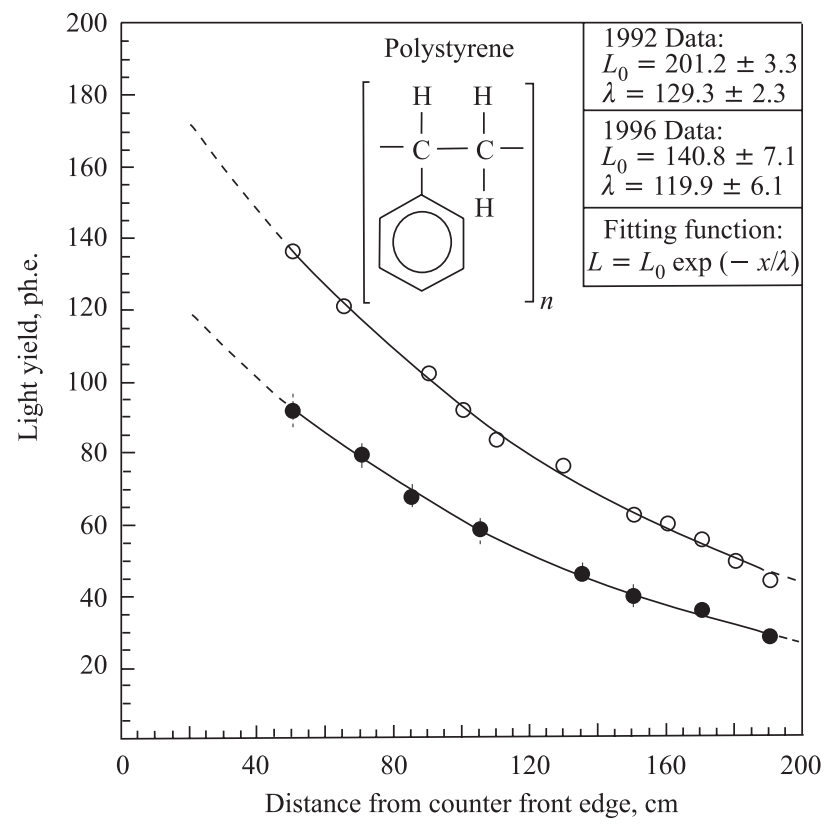

Fig. 11. Light yield of the PS-based scintillator (UPS 923A) counter versus the distance from the front end of the bar. Measurements were done in 1992 (०) and 1996 (॰)

The counter light yield measurements were done in December 1992 and November 1996. The results are summarized in the following figures. In Figs. 11 and 12 we give the light yield as a function of the distance from the front end of scintillator bars for the UPS 923A and NE 114 counters, respectively. For comparison we fitted the corresponding light yield with a single exponential function $L=L_{0} \mathrm{e}^{-x / \lambda}$. As one can see in Figs. 11 and 12, over a period of four years the UPS 923A scintillator light yield decreased by $\approx 30 \%(\approx 7.5 \% / \mathrm{y})$, while the NE 114 scintillator light decreased by $\approx 70 \%(\approx 18 \% / y)$. Thus, natural degradation of the light yield of the NE 114 scintillator bar is more than two times faster than that of the UPS 923A scintillator bar. 
94 Artikov A. et al.

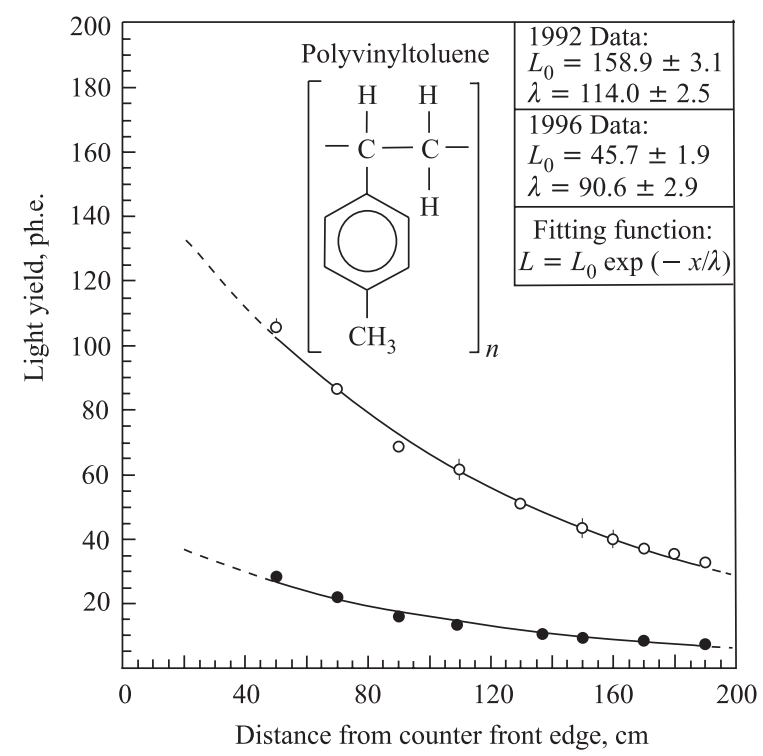

Fig. 12. Light yield of the PVT-based scintillator (NE 114) counter versus the distance from the front end of the bar. Measurements were done in 1992 (०) and $1996(\bullet)$

Visual inspection revealed that the NE 114 scintillator bar got a yellow-green color in contrast to the Ukrainian UPS 923A scintillator bar which remained quite blue. The ensuring investigation led to the discovery that over the eight-year period the NE 114 scintillator had deteriorated to the degree that when exposed to daylight, the scintillator emitted light visibly shifted to the green, and measurements revealed that the transmission had indeed decreased by $1 \%$ per $\mathrm{cm}$ between 400 and $500 \mathrm{~nm}$ where most of the scintillator emission occurs [2].

The polymeric bases of the PVT and PS scintillator bars have different chemical structure. This difference is determined by the fact that in PVT, unlike the case in PS, a methyl group $\left(-\mathrm{CH}_{3}\right)$ substitutes for one hydrogen atom in the benzene ring (see Figs. 11 and 12).

Processes resulting in both chemical and physical structure degradation occur in the polymeric base with time. It is manifested in appearance of dotty defects on the surface and in the bulk of the scintillator bars, as well as microvoids, cracks known as «silver» and minute cracks.

Originally, three types of free radicals are possible to appear in PVT under the effect of external destabilization factors such as temperature, UV light, oxygen, humidity and external radiation:

1) a tertiary methylbenzene radical is created by tearing off the tertiary atom of hydrogen;

2) a primary benzene radical is created by tearing off a hydrogen atom from the methyl group;

3) a benzene radical is created by tearing off the methyl group.

In the PS case only one type of free radicals is originally generated - a tertiary benzene radical resulting from tearing off the tertiary atom of hydrogen.

As known, each of the free radicals created under effect of oxygen and other external factors can initiate the polymeric degradation process, in particular the oxidation chain process. 
Hence the probability for degradation processes to begin and their velocity in PVT will be several times higher than in PS, and this difference was for the first time observed by us as described above.

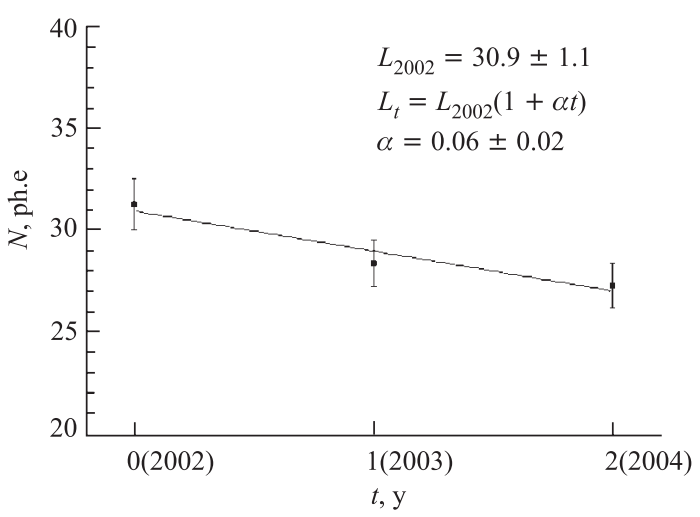

Fig. 13. Dependence of the light yield in terms of the photoelectrons per MIP on time for one of the CSP L3 counters

We also investigated the light yield deterioration of the $320-\mathrm{cm}$-long counter with the WLS fiber readout [2] for the period of 2001-2004. Figure 13 shows dependence of the light yield in terms of photoelectrons per MIP on time. The signal was measured $30 \mathrm{~cm}$ away from the front end of the scintillator counter. In order to survey the long-term stability of the observed light yields, they were fitted as a linear function of time

$$
L=L_{0}(1-\alpha t)
$$

Here $L_{0}$ is the fit parameter which corresponds to the observed light yield at the time of the first measurement and $\alpha$ is the variation rate of the observed light yield. The change in the observed light yield was estimated at a level of about $6 \%$ per year.

In order to evaluate the natural degradation of light output of the scintillator UPS 923A, we performed parallel light yield measurements with a small sample over a 12 -year period. The scintillator sample was a disk $16 \mathrm{~mm}$ in diameter and $10 \mathrm{~mm}$ thick with polished surface. The sample of the scintillator was stored in a dark place over the entire investigation period. The light yield measurements were carried out by using a radioactive ${ }^{137} \mathrm{Cs}$ source for excitation. The sample was placed directly (without optical grease) at the center of the EMI 9814B PMT window and the pulse height conversion electron spectrum of ${ }^{137} \mathrm{Cs}\left(E_{e}=624 \mathrm{keV}\right)$ was measured. Light yield is measured relative to a reference sample whose light output was calibrated in percent of anthracene. The results of our investigation are presented in Fig. 14 We did not observe natural degradation of the scintillator UPS 923A light output over the 12-year period.

The scintillation efficiency (light output) degradation is first of all defined by the degree of luminescent dopant (LD) damage. The LDs used in the UPS 923A scintillator were POPOP and PTP, which have much smaller thermal and thermo oxidation destruction than the PS base [24]. 
96 Artikov A. et al.

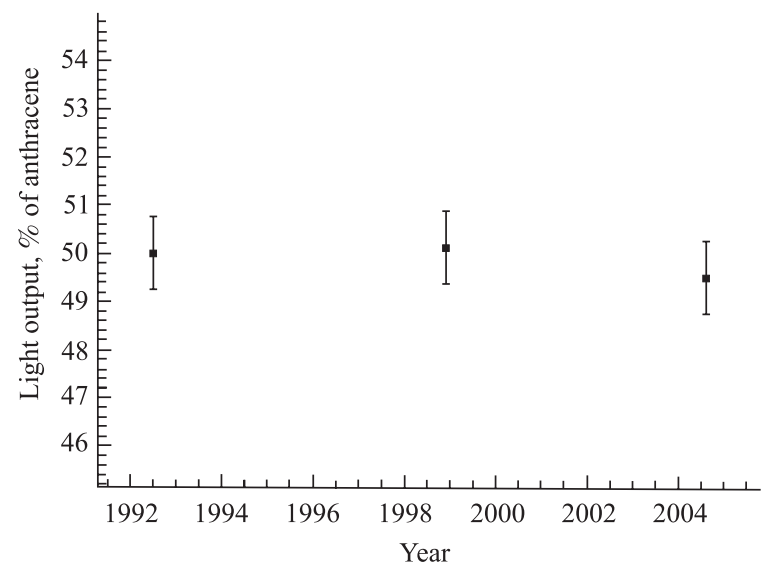

Fig. 14. Light output of a small sample in percent of anthracene as a function of time

In conclusion, note that natural scintillation aging is mainly defined by destruction of the polymeric base.

\section{PRODUCTION OF SCINTILLATOR COUNTERS}

Above 600 muon scintillation counters were built at JINR for the CDF II CSP and BSU hodoscopes. Although shapes and dimensions were different, the same light collection method as shown in Fig. 2 was applied to all counters.

The final design dimensions of the CSP and BSU counters are listed in Table 2.

Table 2. Dimensions of the new scintillator bars

\begin{tabular}{|l|c|c|c|}
\hline Type & Length, $\mathrm{mm}$ & Height, $\mathrm{mm}$ & Thickness, $\mathrm{mm}$ \\
\hline CSP L1 & 2400 & 320 & 20 \\
CSP L2 & 3100 & 320 & 20 \\
CSP L3 & 3200 & 320 & 20 \\
BSU & 1640 & 166 & 15 \\
\hline
\end{tabular}

Three different types of CSP counters were needed to cover parts of the central detector with different space restrictions. Long counters (CSP) were $2 \mathrm{~cm}$ thick. The BSU bars, whose length did not exceed $163.8 \mathrm{~cm}$, were made $1.5 \mathrm{~cm}$ thick. Prototype tests have shown that this was sufficient to ensure efficient counter performances.

A small notch was machined on one corner of each of the scintillator bars to house the PMT (Fig. 2). The area occupied by the notch is only a few tenths of a percent of the total scintillator area, causing only a very small reduction in the geometrical acceptance of the counter. Flat WLS ribbons for light collection along the long narrow edge of the scintillator bar were made by gluing 15 or 20 (depending on counter thickness) 0.1 -cm-diameter fibers with optical cement (BC 600) directly on the scintillator bar edge. The fiber ribbon protruding 
into the notch was shaped into a bundle and the bundle was glued inside an adaptor. The ends protruding from the adaptor were cut flat and the surface was ground and polished as required for good quality of optical contact with the PMT photocathode. In order to maximize light collection the ribbon ends farthest from the PMT were «mirrored». This was accomplished by grinding the flat ribbon end with sand paper and gluing a small mirror.

All faces of the scintillator bars, except the one carrying the WLS fiber ribbon, were accurately polished. Aluminum foil strips were laid on the narrow side opposite to the one carrying the ribbon so as to reflect the escaping light back into the scintillator. A lightreflecting aluminum strip was glued by optical glue to the outer side of the WLS ribbon for increasing the light capture efficiency.

The large-area bar faces were covered with «orange skin» aluminum-backed paper for reflection of the light exciting the scintillator. These faces were backed by black plastic for light tightness.

Blue light is emitted by the POPOP wavelength shifter in the scintillator. This light reaching the fiber ribbon is absorbed by the Y11 (K27 formulation) shifter with subsequent emission in the green region of the spectrum. Part of green light propagates by total internal reflection in both directions along the fibers. The mirror located on the far edge reflects the light back towards the PMT, greatly increasing the light collection efficiency [6-10].

Multi-clad S-type WLS Y11 (250 ppm) fibers produced by Kuraray (Japan) were used for most of the WLS fiber ribbons. The fiber core is polystyrene with the Y11 shifter, the inner cladding is polymethylmetacrylate (PMMA) and outer cladding is fluorinated PMMA.

Pol.Hi.Tech (Italy) K27 (200 ppm) fibers were used for about $30 \%$ of the BSU counters.

It should be noted that the light capture efficiency in multi-clad fibers $(\approx 5.35 \%)$ is significantly superior to that of single-clad fibers $(\approx 3.14 \%)$. This factor was the most significant one in bringing the light collection efficiency of WLS fibers into the range which made them useful for the application described here.

The 2.2-cm-wide, 2.2-cm-high and 6-cm-long H5783 photosensor manufactured by Hamamatsu Photonics, was used as a photon detector. The effective diameter of the photocathode is $0.8 \mathrm{~cm}$ and its quantum efficiency is $\approx 12 \%$ at the average wavelength of the Y11 (or K27) emission spectrum $(\approx 500 \mathrm{~nm})$.

The counters were subjected to strict quality control during production. It included check of the precision in the mechanical dimensions, quality of gluing, quality of surface polishing, light shielding and, finally, robust packing for safe shipping. The importance of very careful gluing of the fragile WLS fibers deserves a special mention. Air bubbles must be avoided in gluing because they seriously degrade light collection. The detailed assembly procedure of counters is described in [25].

The completed counter was thus ready to be tested with cosmic muons and radioactive sources.

\section{COUNTER PERFORMANCE AND QUALITY CONTROL}

The investigation of detector performance and quality control were carried out by using cosmic muons. The block diagram of a measurement setup is shown in Fig. 15. The analog signal from the PMT was amplified by a Model 777 «Phillip Scientific» high-speed amplifier 
( $\approx 100 \mathrm{MHz}$ band width) and measured by an ADC (Le Croy 2249A). The width of the gate signal was $\approx 80 \mathrm{~ns}$.

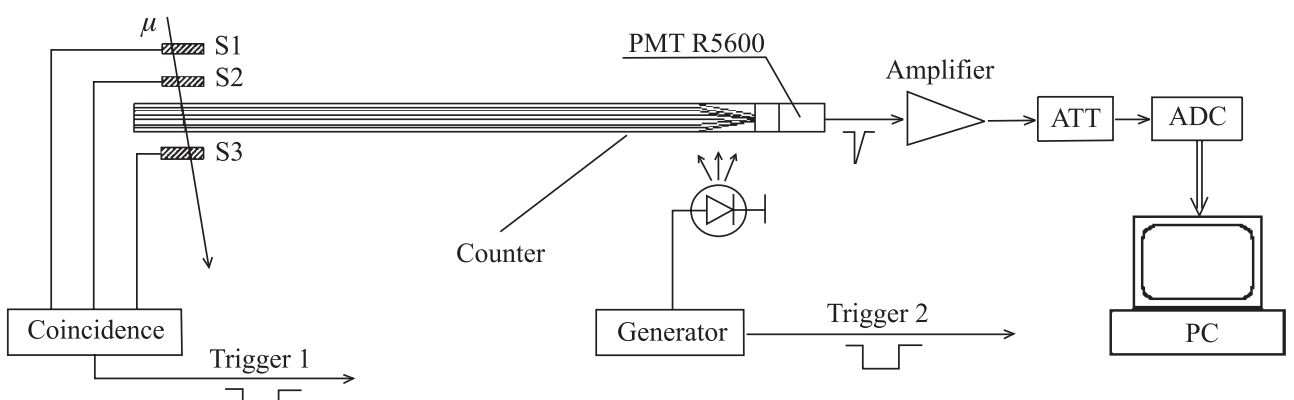

Fig. 15. Block diagram of the setup used to test the counters

The PMT signal was suitably amplified to obtain a single photoelectron spectrum of the amplitude adequate for calibration of a spectrometric channel. An attenuator was used to increase the dynamic range of the measured signals. The ADC output was read out and processed by a PC. The counter test setup was operated in two regimes with different triggers. Trigger 1 was used to measure the cosmic muon spectra (cosmic muons selected by a telescope of three small $7 \times 7 \mathrm{~cm}$ scintillation counters $\mathrm{S} 1, \mathrm{~S} 2, \mathrm{~S} 3$ in coincidence) and trigger 2 was used for the spectrometric channel calibration.

The counter to be tested was sandwiched between S1 and (S2, S3). Moving the telescope along the counter axis, we measured the light yield dependence on the distance from the bar edge. Trigger 2 was provided by a pulse generator and was used for the measurement of the LED spectra. The fast AlGaAs LED HLMP8100 (Hewlett Packard) was driven by a pulse generator with short pulse width $(\approx 10 \mathrm{~ns})$. The photon flux incident on the PMT photocathode was tuned by varying the supply voltage to the LED. The LED spectra were used to determine the spectrometric channel parameters and to monitor their overall time drift. For these purposes LED spectra were measured before and after each cosmic muon run.

The spectrometric channel was calibrated by measuring, in absolute units, the distribution of the number of photoelectrons emitted by the PMT photocathode for a traversing cosmic muon.

The light yield in absolute units characterizes the counter performance. In principle, it would be sufficient if it were at least as large as needed to obtain full efficiency, but in practice, it is important that it is substantially larger to accommodate degradation with time. The knowledge of light yield in absolute units is very important as it enables one not only to find the efficiency of the counters and to compare parameters of different detectors, but also to estimate the long-term stability of the counters $[10,11]$.

The details of this calibration method can be found in our work [12]. The yield dependence on the longitudinal coordinates was measured with cosmic muons which traversed the cosmic telescope to trigger data acquisition. During this measurement the ADC dynamic range was adjusted as convenient by varying the attenuator. Runs about two hours long were needed for each measurement in order to reach the sufficient statistical precision $(\approx 4 \%)$. 


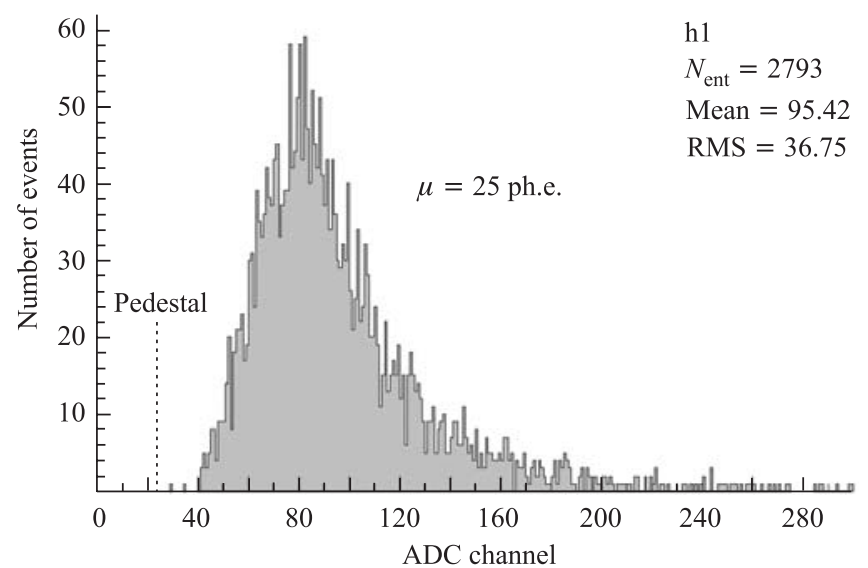

Fig. 16. Typical cosmic muon spectrum taken from the far end of the CSP L3 counter

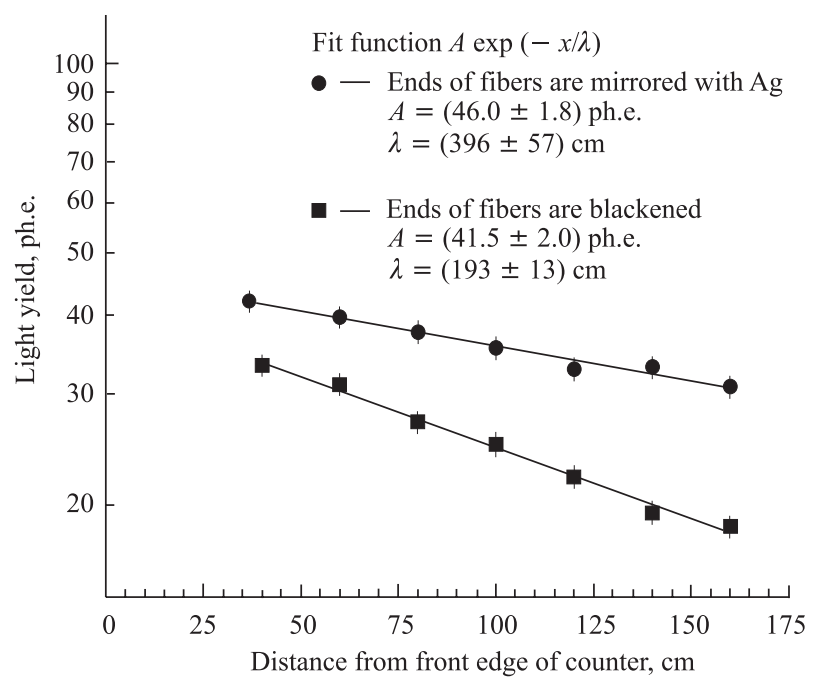

Fig. 17. Measurements of light yield of a BSU counter (15 WLS fibers, Kuraray)

A typical cosmic muon spectrum of a CSP L3 counter is shown in Fig. 16. The dashed line represents the pedestal centroid. From this spectrum we find the average muon signal in photoelectrons. The attenuation curve for one of the BSU counters is presented in Fig. 17. The light reflection coefficient from the mirrored fiber end is more than $70 \%$. Correlation between the light yield from the far end for one lot of BSU counters and their BAL values (see Fig. 5) is presented in Fig. 18. One can see that light yield is less dependent on the BAL for counters with the WLS fiber readout.

All the BSU and CSP counters were tested as described above. Figure 19 shows the distribution of photoelectrons over the entire sample for muons traversing close to the far end of the counters. 
100 Artikov A. et al.

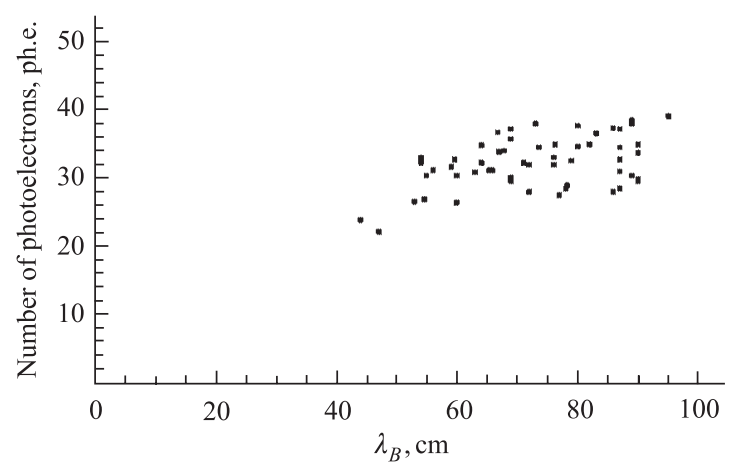

Fig. 18. Correlation between the light yield from the far end for one lot of the BSU counters and their BAL values
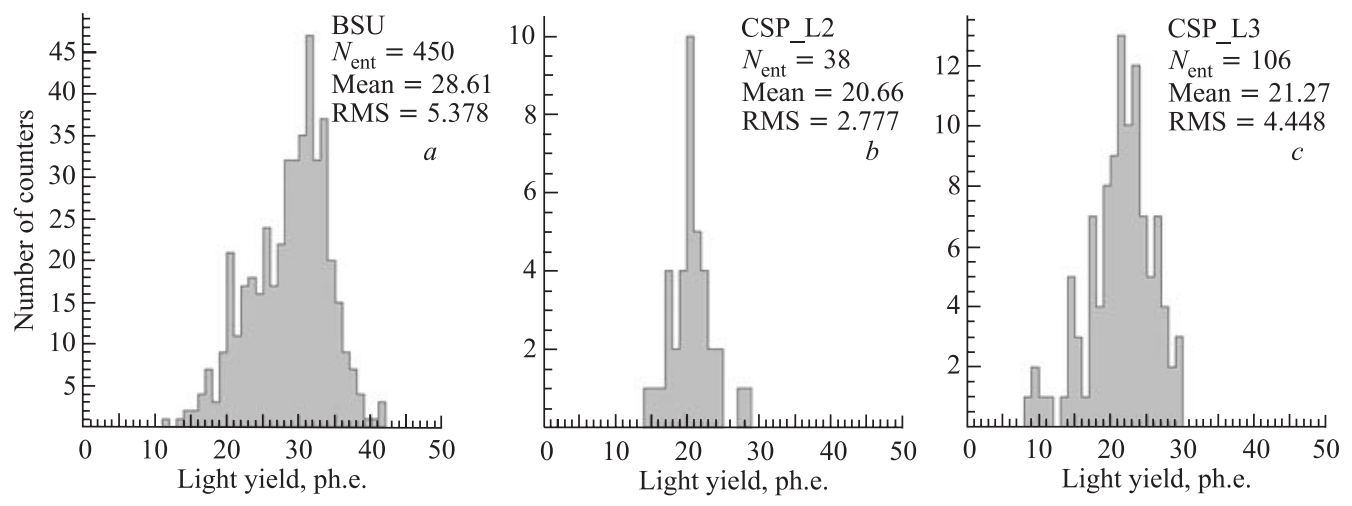

Fig. 19. Distribution of the counter light yield from the far end for the BSU and CSP counters

Note that all tests of the counters were made without optical grease between the fiber bundle and the PMT face. Using optical grease we can increase the light yield of the counters at least by $\approx 10 \%$.

\section{CONCLUSION}

A novel technique for collecting light from large-area scintillation counters has been developed and successfully used for construction of about 600 new counters ranging from 160 to $320 \mathrm{~cm}$ in length for the CDF muon upgrade. The technique relies on wavelength shifter fibers to extract light from the longer side of the scintillator bar, thereby reducing the path length of the light in the bulk material and consequently the importance of good light transmission in the counter. Performance of long bars is therefore less dependent on the scintillator transmission properties and less susceptible to its deterioration as compared to conventional light guides. 
Another important advantage of this technique is the reduced cross section of the fiber bundle, which allows one to use a smaller-area phototubes. Elimination of lucite light guides and large photomultipliers results in a much more compact design for which the ratio of sensitive to total area is close to one. The reduced sensitivity of small photomultipliers to magnetic fields can also be a significant advantage.

The Ukrainian UPS 923A scintillator used to construct the counters was polystyrenebased plastic developed by the Monokristal Institute in Kharkiv under the supervision of JINR (Dubna). The counters were constructed at JINR by using a cost-effective technique developed in collaboration with INFN/Pisa and engineered at JINR.

The results of quality control tests performed at JINR by using the same photomultiplier for all counters show that the average light output ranges between 21 ph.e./MIP (for the longer counters) and 28 ph.e./MIP (for the shorter ones) for muons traversing the counters normally at the farthest ends from the photomultipliers. The light collection efficiency obtained is more than adequate for the $100 \%$ detection efficiency over the entire counter area. In view of a tolerant deterioration rate of $5-10 \%$ per year, full efficiency should be retained for the useful lifetime of the CDF.

The rise time of the light pulse of the UPS 923A scintillator is less than $0.8 \mathrm{~ns}$ and the rise time of the light pulse of the «UPS 923A plus WLS fiber» system is about 2 ns. Therefore, scintillation counters with the WLS fiber readout can be used in fast coincidence systems to observe rare particles against large background of other events and in time-of-flight measurements.

The method for measurements of the Bulk Attenuation Length of the scintillator for its own light emitted was proposed. It was found that the BAL of the UPS 923A scintillator for its own light emission is not a constant value and strongly depends on light propagation in the scintillator due to reabsorption effect.

It is essential to note that the eventually chosen Kharkiv (Ukraine) polystyrene-based scintillator has excellent properties adequate for the long-term experiments unlike the scintillator with polyvinyltoluene base.

\section{REFERENCES}

1. The CDF-II Detector Technical Design Report. The CDF II Collaboration. Fermilab-Pub-96/390-E. 1996. Ch. 10

2. Cabrera S. et al. Making the most of aging scintillator // Nucl. Instr. Meth. A. 2000. V. 453. P. 245.

3. Artikov A. et al. The «Miniskirt» Counter Array at CDF II // Part. Nucl., Lett. 2002. No. 5[114]; http://www1.jinr.ru/Pepan_letters/panl_5_2002/03_art.pdf

4. Bromberg C. // DPF 2000, Ohio, Aug. 2000. Intern. J. Mod. Phys. 2001. V. 16, Suppl. 1C. P. $1143-$ 1146.

5. Freeman J. et al. The CDF Upgrade Calorimeter // Proc. of the Second Intern. Conf. on Calorimetry in High Energy Physics / Ed. A. Ereditato. World Scientific, 1993.

6. Budagov J. et al. IMU Trigger Scintillators: BSU Prototype Results. CDF Note 4210. 1997.

7. Budagov J. et al. CMU Trigger Scintillators: CSP Prototype Results. CDF Note 4211. 1997. 
102 Artikov A. et al.

8. Budagov J. et al. IMU Trigger Scintillators: WSU-TSU Prototype Results and Summary of All IMU Prototype Results. CDF Note 4254. 1997.

9. Budagov Yu. et al. Long Scintillation Counters with the Shift-Spectrum Fiber Light Guides. JINR Preprint P13-93-304. Dubna, 1998 (in Russian).

10. Budagov Yu. et al. Test of Long Scintillating Counter Prototypes for CDF II. JINR Preprint E132000-127. Dubna, 2000.

11. Bellamy E. H. et al. Test of long scintillation counters for large supercollider detectors // Nucl. Instr. Meth. A. 1994. V.343. P. 484-488.

12. Chirikov-Zorin I. et al. Method for precise analysis of the metal package photomultiplier single photoelectron spectra // Nucl. Instr. Meth. A. 2001. V.456. P.310-324.

13. Kuraray Co., LTD. Methacrylic Resin Division. Scintillation Materials. http://www.kuraray.co.jp/en/; e-mail: psf@kuraray.co.jp

14. Kettenring $G$. Measurement of the reflectivities and absorption lengths at different wavelengths of plastic scintillator and acrylglass // Nucl. Instr. Meth. 1975. V.131. P.451-456.

15. Lyuminescentnij Analiz. Fizika i tekhnika spektral'nogo analiza (Biblioteka inzhenera). 1961 (in Russian).

16. Zaidel' A. H., Ostrovskaya G. V., Ostrovskii Yu. I. Tekhnika i praktika spektoskopii. Fizika i tekhnika spektral'nogo analiza (Biblioteka inzhenera). M.: Nauka, 1976 (in Russian).

17. Destruel P. et al. A new plastic scintillator with large stokes shift // Nucl. Instr. Meth. A. 1989. V. 276. P. 69-77.

18. Kirkby J. Today and Tomorrow for Scintillating Fibre (SCIFI) Detectors. CERN-EP/87-60. 1987.

19. Gioromini P. et al. The Central Muon Extension Scintillator (CSX). CDF Note 3898. 1996.

20. Bollinger L.M., Thomas G. E. Measurement of the time dependence of scintillation intensity by a delayed-coincidence method // Rev. Sci. Instr. 1961. V.32. P. 1044.

21. Birks J. B. Fluorescence response functions and scintillation pulse shapes // J. Phys. B. 1968. V. 1. P. 946.

22. Matveeva E.N. et al. Measurements of timing properties of the fluorescence fast component from plastic scintillator // Nucl. Instr. Meth. 1981. V. 179. P. 277.

23. Bellamy E. H. et al. Absolute calibration and monitoring of a spectrometric channel using a photomultiplier // Nucl. Instr. Meth. A. 1994. V. 339. P. 468-476.

24. Senchishin V.G., Verezub N.B., Lavrienko S.N. Tekhnologia proizvodstva polimernykh opticheskikh izdelij. Kiev, 1992. S. 68 (in Russian).

25. Artikov A. et al. Design and construction of new central and forward muon counters for CDF II // Nucl. Instr. Meth. A. 2005. V.538. P. 358-371. 